\title{
A Systematic Review of Clinical Decision Support Systems in Alzheimer's Disease Domain
}

\author{
https://doi.org/10.3991/ijoe.v17i08.23643 \\ Sherimon P.C. ${ }^{1}$, Vinu Sherimon $\left.{ }^{2}{ }^{凶}\right)$, Preethii S. P. ${ }^{3}$, Rahul V. Nair ${ }^{4}$, Renchi Mathew ${ }^{4}$ \\ ${ }^{1}$ Arab Open University, Muscat, Sultanate of Oman \\ ${ }^{2}$ University of Technology and Applied Sciences, Muscat, Sultanate of Oman \\ ${ }^{3}$ Government Vellore Medical college, Tamil Nadu, India \\ ${ }^{4}$ Royal Oman Police Hospital, Muscat, Sultanate of Oman \\ vinusherimon@yahoo.com
}

\begin{abstract}
Dementia is one of the major public health issues faced by the world. Alzheimer's disease (AD) is the most common form of dementia targeting old age groups around the world. It is a neurodegenerative condition with memory loss as its early symptom. Unfortunately, there is no cure for this disease currently. So various research in the medical and technical fields are being conducted to help people with Alzheimer's. Many studies focus on early diagnosis of Alzheimer's disease using clinical decision support system (CDSS) so that the progression of the disease can be slowed down to a great extent. In this context, we have undertaken a research to design and implement an ontology based Clinical decision support system for Alzheimer's disease in Sultanate of Oman. A semantic knowledgebase (ontology) will be the core component of our Clinical decision support system. The objective of this research paper is two-fold (a) review the medical aspects of Alzheimer's disease, and (b) review the available clinical decision support system based on ontology, robotics, and mobile applications in Alzheimer domain. Research articles published during 2011- 2020 in PubMed, Google scholar, Elsevier, SpringerLink and IEEE journals were reviewed. We found that there are various clinical decision support systems which can aid physicians in suggesting diagnosis, and treatment of Alzheimer's disease.
\end{abstract}

Keywords-dementia, Alzheimer's disease, clinical decision support system, ontology, knowledge base, memory loss, mental deterioration, mental disorder

\section{Introduction}

Dementia is a syndrome associated with persistent deterioration in brain function. It can affect memory, thinking, and other mental abilities [1]. Alzheimer's is the most common cause of dementia affecting millions of people around the world.AD is a neurodegenerative disease usually presented in older people who are 65 years of age and more. It causes neuronal cell death resulting in cerebral atrophy and widening of third ventricle of brain[2]. Depending on the cognitive impairment, AD can be classified into mild, moderate, and severe. The early signs and symptoms of AD includes 
(a) Memory loss that disrupts daily life (b) Trouble with planning or solving problems, (c) Difficulty in completing familiar tasks, (d) Confusion over time or place, (e) Problems with understanding visual images and spatial relationships, (f) New problems with words when speaking or writing, (g) Misplacing things, (h) Poor judgement, (i) Dismissal from work or social activities, (j) Mood and personality changes [3]. There is no cure for Alzheimer's disease currently, but medications are available that can reduce the symptoms temporarily.2 Treatment includes Cholinesterase inhibitors and Partial N-Methyl D-aspartate (NMDA) antagonist [2]. Many AD projects indicate that neuroplasticity can help AD patients.

Clinical Decision Support Systems (CDSS) are systems that supports the medical practitioners with the knowledge and patient specific data to have accurate diagnosis and to improve the quality of health care. Many health organizations have implemented CDSS for different types of diseases. One of the major challenges in today's health domain is successful transfer of health data and its unique interpretation across different health information systems. When a patient data is transferred from one hospital to another, it is valuable, if the concerned practitioner's in both the hospitals arrive at the same conclusion. Otherwise, it may lead to wrong understandings and implications, which is very critical in health domain. Also, the data available from different sources must adapt a common specification, such as data context, data structure and the meaning of the data. So, a semantic interoperability must be achieved to preserve the context and meaning of the data. The "knowledge" itself must be shared to arrive at the same interpretation. Ontologies are best for semantically representing the knowledge. It is defined as a formal, explicit specification of a shared conceptualization. To develop efficient clinical decision support systems, Ontologies can be used to integrate the patient health record with data from different sources. Ontologies manage and specify the knowledge from the medical domain.

\subsection{Research questions}

RQ1: What is Alzheimer's disease and how does it affect people? Mention Alzheimer's disease's aetiology, epidemiology, pathophysiology, genetic origin, medical background, clinical exams, clinical characteristics, laboratory investigations, and management.

RQ2: What is neuroplasticity and what function does it serve in Alzheimer's disease treatment?

RQ3: In Alzheimer's disease, what part does a clinical decision support system play?

RQ4: What function does ontology perform in Alzheimer's disease?

RQ5: Are there any ontology-based clinical decision support systems for Alzheimer's disease?

\section{Methodology}

The increasing amount of research makes it more difficult for practitioners and researchers to stay abreast of previous and current results within and across disciplines. 
As a result, research interests do not draw on or promote past findings, but rather exist independently of the larger amount of knowledge. Since the sample collection of appropriate studies influences a review's result, validity, and persuasive force, vigorous evidence detection is necessary for systematic reviews. To this day, it is uncertain which search systems are best for systematic reviews and why. There is no guidance on which search engines and bibliographic databases to use for structured searches, and there are no comprehensive, scientific performance evaluations.

The first aim of a systematic review is to locate all or as many applicable tools as possible. As a result, the reviewer must choose a search system that offers the most comprehensive coverage of the chosen search subject. Taking into consideration that ours is an interdisciplinary research, an extensive survey was conducted to identify the relevant journal articles. The research articles published from 2011 to 2020 that represent the symptoms, stages, causes, treatments of AD and the application of Ontology and CDSS in Alzheimer's domain were included in the study. The most popular search engines such as PubMed/Medline, Science Direct, Scopus, IEEE and Google Scholar were used to find the related resources. PubMed search engine was found more suitable to find the resources related to AD as it provides high coverage of literature from biomedical and life sciences domain.

Articles were searched using the key words: "CDSS", "Ontology" and "Alzheimer's Disease". And the Boolean operator "AND" was used for accurate search results. The returned results included the applications of (a) Ontology in Alzheimer's domain, (b) CDSS in Alzheimer's domain, and (c) Ontology based CDSS in Alzheimer's domain.

Twenty-five research articles published between January 2011 and September 2020 in English language are included in this literature review. The articles published before 2011 were excluded in the literature survey. Initially, during the search 615 articles were retrieved and 128 were excluded for not meeting the criteria -Year of publication. Among the remaining 487 articles, 456 of them were excluded due to various reasons such as studies not relevant to the main topic, non-availability of full texts, etc. Out of the remaining 31 articles, 6 were excluded because of repetition. Figure 1 shows the flowchart of the search process of the literature.

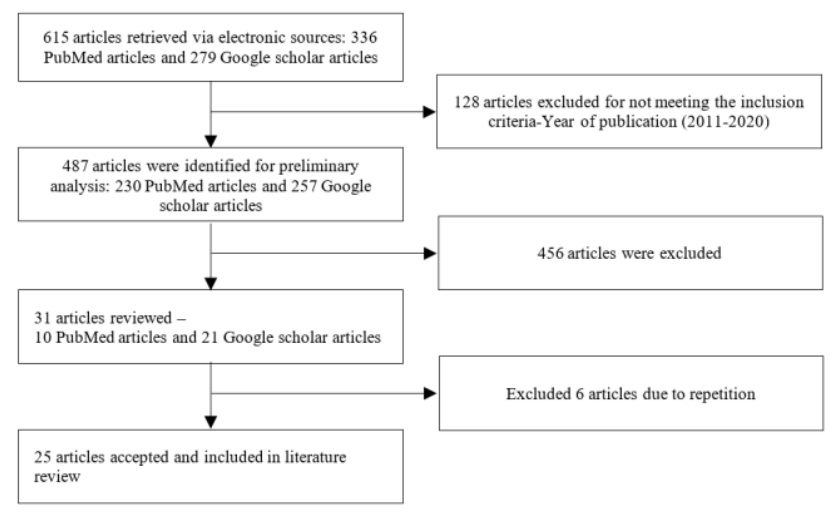

Fig. 1. Flow diagram of literature screening process 


\section{Results and Discussion}

3.1 RQ1: What is Alzheimer's disease and how does it affect people? Mention Alzheimer's disease's aetiology, epidemiology, pathophysiology, genetic origin, medical background, clinical exams, clinical characteristics, laboratory investigations, and management?

Alzheimer's disease (AD), the most common type of dementia worldwide. It is a neurodegenerative condition usually presented in the older people at the age of 65 years and above and is the sixth leading cause of death in the United States [2].

Aetiology: The exact cause of Alzheimer's is not clear, but the risk factors associated with $\mathrm{AD}$ are older age, family history of dementia, head trauma, depression, diabetes, hypertension, heart diseases, cerebrovascular diseases, smoking, physical inactivity, trisomy21, presence of APOE e4 allele, high homocysteine level [4].

The pie chart in Figure 2 demonstrates the Alzheimer population and their respective age group in the US in 2020 [5]. 1 million people between 65 to 74 years of age, 2.7 million people between 75 to 84 years old and 2.1 million greater than 85 years old were affected by Alzheimer's disease in the USA in 2020.

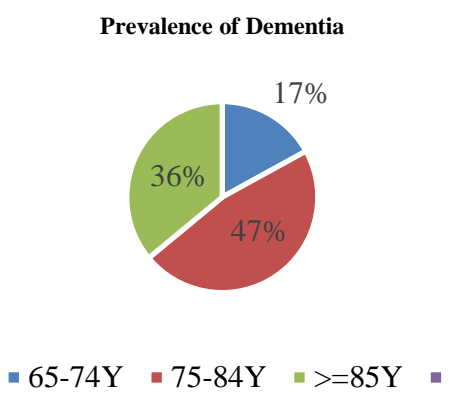

Fig. 2. Alzheimer population and their respective age group in the US in 2020

There are certain things that can reduce the risk or delay the onset of dementia. This includes higher education, oestrogen use by women, anti-inflammatory drugs, reading books, playing musical instruments, healthy balanced diet, aerobic exercise [4].

Epidemiology: 50 million people around the world suffer from dementia. Alzheimer's patient occupies $60-70 \%$ of this dementia population [6]. For every $67 \mathrm{sec}-$ onds someone in the United States develops AD [7]. Prevalence of dementia in the Arab countries ranges about (a) $1.1 \%-2.3 \%$ in 50 years of age and above, (b) $13.5 \%$ $18.5 \%$ in 80 years of age and above which is almost same as other countries in the world [8]. Most recent WHO data published in 2018 indicates that Alzheimer's and Dementia death rate in Oman is 397 or $3.86 \%$ of total deaths. Oman ranks 49th in the world with an age adjusted death rate of 32.04 per 100,000 population [9].

It is estimated that dementia population around the world will be 50 million, 82 million and 152 million in 2019, 2030 and 2050, respectively (Figure 3). 


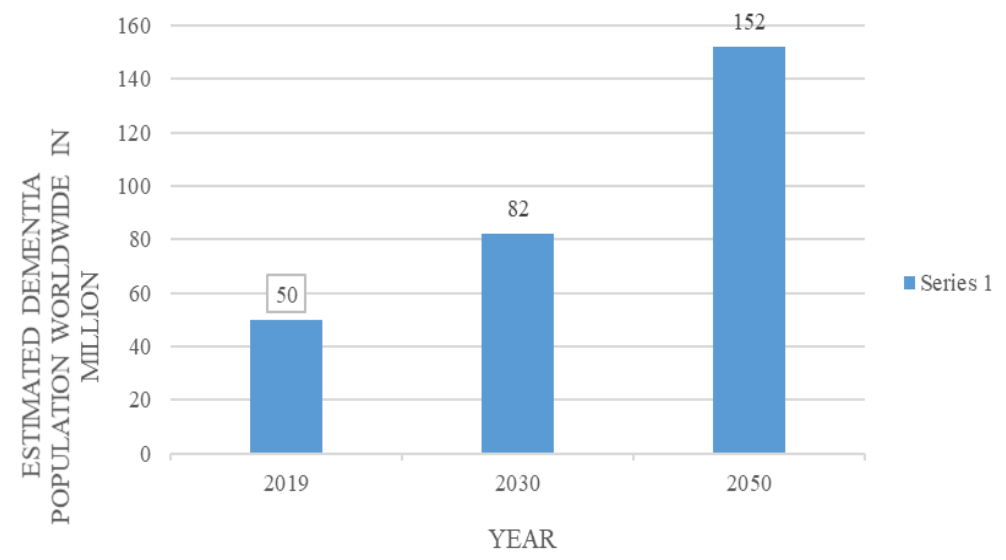

Fig. 3. Estimated dementia population around the world in 2030 and 2050 [10]

Pathophysiology: Neuronal cell death due to the accumulation of abnormal neuritic plaques and neurofibrillary tangles results in AD. Plaques are extracellular amyloid $\beta$ peptide. Neurofibrillary tangles are intracytoplasmic fibrillary structures inside the neurons made up of tau protein [2]. Initially neuronal cell death occurs in hippocampus, the part of the brain necessary for forming memories [11].

Trisomy21 causes Down syndrome. Over expression of amyloid precursor protein gene in the chromosome 21 produces $\beta$ amyloid protein which increases the risk of $\mathrm{AD}[12]$.

Genetic basis of ad: Three genes are associated with the autosomal dominant form of AD. They are AAP gene-chromosome 21, Presenilin1- chromosome 14, and Presenilin2-chromosome 1.

History and physical examination: Getting a complete history from a reliable source (family member/caregiver) is mandatory. Complete physical examination, neurological examination and mental status examination is a must to diagnose AD and to rule out all other causes.

Clinical features: Depending on the cognitive impairment, AD can be classified into mild/ early, moderate, and severe [2]. Signs and Symptoms of AD depends on the stage of the disease. Short term memory loss, trouble with solving problems, judgment, and executive function; lack of motivation, disorganization, problems with multitasking in the early stage of AD. Followed by language disorder and visuospatial skills impairment in the moderate stage. Neuropsychiatric symptoms, dyspraxia, olfactory dysfunction, sleeping disturbances, extrapyramidal motor signs in the late stage. Finally, primitive reflexes, incontinence, and complete dependence on caregivers [2].

Laboratory investigations: Routine lab investigations such as complete blood count, complete metabolic panel and thyroid stimulating hormone levels are checked to rule out other causes. CT brain imaging may show atrophied cerebrum and widened third ventricle. Cerebrospinal fluid indicates low $\beta$ amyloid 42 and increased tau protein level. Genetic testing can be done to detect abnormal genes [2]. Figure 4 displays the MRI of brain showing the brain atrophy pattern in various sub types of AD. 


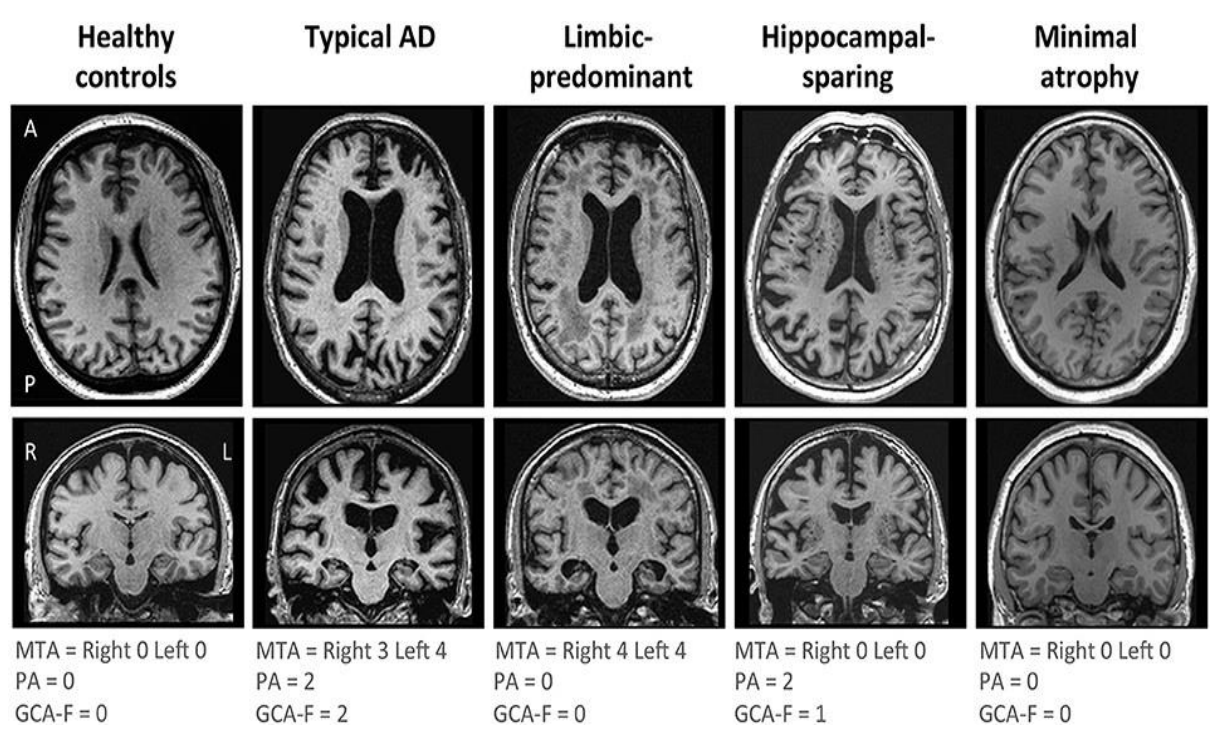

Fig. 4. MRI Brain showing brain atrophy pattern in various sub types of AD [13].

Management: There is no cure for Alzheimer's disease currently. Medications for AD may temporarily improve symptoms or slow the disease progression. This treatment can also help people with Alzheimer's to improve function and maintain independence for a period [14]. Treatment includes Cholinesterase inhibitors such as Donepezil, galantamine and Rivastigmine and Partial N-Methyl D-aspartate (NMDA) antagonist Memantine.

Alzheimer's disease is a neurodegenerative disease-causing neuronal cell death. Abnormal neurotic plaques and tangles accumulate in the neurons leading to damage and destruction of these cells which leads to dementia in old age population. CT scan may show atrophied cerebrum and widened third ventricle. At present, this disease is managed with medications to improve the symptoms and to slow the progression. Aerobic exercise, playing musical instruments, memory tasks, yoga, learning new language can induce plasticity which can compensate the neuronal cell damage caused due to ageing/injury to some extend in Alzheimer's patient.

\subsection{RQ2: What is neuroplasticity and what function does it serve in Alzheimer's disease treatment?}

Neuroplasticity: Adaptation of brain cells in response to a lifetime's experience is termed as neuroplasticity. And it is believed to be maintained to some extend throughout the aging process. Aerobic exercise and cognitive stimulating activities like playing musical instruments can induce plasticity which can compensate the brain damage due to ageing/injury to some extent [15]. Interventions increasing neuroplasticity can bring positive cognitive gains particularly people in risk populations or mild disease. Transcranial magnetic stimulation and transcranial direct current stimulation can cause a long-lasting significant modification in neuroplasticity of the brain [16]. 
Dementia care givers: Dementia is a major problem faced by countries all over the world, with mental and socioeconomic impacts on caretakers and their family [17]. Alzheimer's symptoms often appear slowly. This can start when you find it difficult to remember what just happened, or you are unable to express your thoughts in words. But over time, the problem gets worse. People in advanced stages of the disease usually cannot live alone or take care of themselves [18]. In the advanced stages they are taken care by caregivers. Dementia caregivers are facing bad consequences in their physical and mental health due to the tiring tasks they do to take care of dementia patients [19].

\subsection{RQ3: In Alzheimer's disease, what part does a clinical decision support system play?}

Clinical decision supporting system (CDSS): Public health problems are increasing as the population continues to grow. Consequently, the amount of data that needs to be processed increases exponentially. Big data tools that can effectively minimize processing time and create error free data are in need [20]. One such development is CDSS (Clinical Decision Support Systems), a computerized program that analyses data from an electronic disease record to provide diagnosis and alerts to help healthcare providers implement an evidence-based clinical advice at the point of care [21]. Based on the information obtained from patient data, CDSS can suggest the most probable diagnosis, [22] and assists physicians with decision making tasks [23]. CDSS is implemented in various medical domain such as dementia, Diabetes, mild cognitive impairment and many more. CDSS is a major player in the medical artificial intelligence field and an innovative method that can empower medicine [20].

Clinical decision support system (CDSS) is a computer-based application that can provide clinical recommendations for medical conditions with the help of electronically derived patient's data such as symptoms, clinical features, clinical examinations, imaging, laboratory tests, genetic tests, neurological examinations, and neuropsychiatric examinations. Various Clinical decision support system in Alzheimer's domain is developed to aid physicians in diagnosis, investigations, staging the severity of the disease.

CDSS in medicine: Role of CDSS in medicine is getting advanced day by day. With the help of medicine domain experts, a Bayesian network based CDSS was developed in 2020 to suggest diagnosis, relevant lab tests and treatment for various diseases using the patient's symptoms. The results were to be promising and it can be used for multiple diseases [24].

CDSS in Alzheimer's disease: In 2012, CDSS for the diagnosis Alzheimer's disease using a Bayesian network and an influence diagram as the decision-making model was created. They designed a Bayesian network with fixed structure and probabilities automatically gathered using a machine learning algorithm called Expectation-Maximization. They used a patient dataset provided by the Consortium to Establish a Registry for Alzheimer's disease (CERAD). The subject database was composed by several forms of information using rating scales which helped in assessing various components of the Alzheimer's disease. Then they evaluated the Bayesian 
network using discrimination and probability performance measurements. Then the results were compared to other classifiers [23].

An experience-based clinical decision support system (CDSS) to diagnose Alzheimer's disease using a set of Experience Knowledge Structure (SOEKS) was created. This method opens new knowledge in the system and creates new rules that stimulate reasoning. An illustrative case of their system was also presented by them [25].

A CDSS based Dementia management and support system (DMSS) was developed to support physicians to diagnose dementia and to suggest relevant investigations. It was implemented in 11 clinics in 4 countries. Results from comparison studies exhibit that the system and the physician comply in $84.6 \%$ of the patient cases [26].

A CDSS which can determine the severity of AD particularly using cognitive and functional assessments was developed. CFA dependent CDSS was developed because of the high accuracy rate of CFA over other diagnostic modalities. Kernel Ridge Regression and Support Vector Machine were implemented in the system. The most excellent SVM performance was once determined for a set of 4 CFA check scores (FAQ, ADAS13, MoCA, MMSE) with multi-class classification accuracy of $83.0 \%$, 95\% CI $=(72.1 \%, 93.8 \%)$. Excellent performance of the KRR method using both CFA and MRI neuroimaging data was found to be $\mathrm{R} 2=0.874,95 \% \mathrm{CI}=(0.827$, 0.922). Hence this CDSS model has more practical applications and accuracy [27].

Generally, CDSS used nowadays are static which are not upgraded when new statics are added. Adding new data can aid in gaining new expertise and increases the accuracy rate. Hence, a dynamic CDSS that can update by itself routinely was developed using supervised machine learning algorithms. Various decision models are ranked according to their performance by evaluating the patient data added to them by hospitals or guidelines. Patient datasets were collected from the Institute if Psychiatry of UFRJ and Antonio Pedro Hospital of UFF. The best selected decision model is them used in CDSS and it was found to be more effective in diagnosing dementia, Alzheimer's disease, and mild cognitive impairment [28].

\subsection{RQ4: What function does ontology perform in Alzheimer's disease?}

Ontology: "An ontology is a formal description of knowledge as a set of concepts within a domain and the relationships that hold between them" [29]. Ontology is considered appropriate for database management system for its potential to create semantic relations through which an informed conclusion is made [30]. Applications of ontology in AI (Artificial intelligence) are investigated in various research projects [31].

\section{Ontology in Alzheimer's disease}

In Tunisia, in the year 2015, they developed fuzzy based ontology for Alzheimer's domain called "AlzFuzzyOnto". It consists of representation and manipulation of information related to the diagnosis and the treatment of the Alzheimer's disease, while taking into consideration of the fuzzy aspects associated with the clinical method of AD. They started with (a) a published version of the Mind ontology, the early code of the Ontology, and (b) developed it with the help of experts in the field, (C) Identify points of uncertainty and ambiguity for each concept and each relationship 
that will arise from fuzzy concepts and fuzzy relationships for representing data and fuzzy knowledge. Several sources of knowledge were scanned and collected from subject matter experts, including "Guideline for Alzheimer's disease Management" and "Maladie d'Alzheimer - Enjeux scientifiques, médicaux et sociétaux" and by domain experts. SNOMED CT UMLS Meta-thesaurus were used to create "AlzFuzzyOnto". And they aim in continuing their research to build a fuzzy rule base consisting of export knowledge and finally a fuzzy inference engine [32].

An ontology-based method to enhance data querying and organization of AD data was done in 2018. ADNI-Alzheimer's Disease Neuroimaging initiative collects data of Alzheimer subjects like clinical exams, imaging reports, genetic and biochemical biomarkers to diagnose $\mathrm{AD}$ at its early stage. By using ontology, ADNI was developed which can access a particular visit of the patient using hasEvent property in it and all the relevant examinations can be retrieved using collects property [33].

In 2020, researchers from Brazil proposed an ontology which can work as a common knowledge base in mental health domain. It can provide information about symptoms, diagnosis, treatment, prevention [34].

The projects taken by various research teams in the field of the Alzheimer's disease and the ontologies they created were analyzed and compared. The aim was focused to provide a standardized information with a common base framework and data recovery in the $\mathrm{AD}[35]$.

A bilingual domain ontology from termino-ontological and textual sources for semantic representation and information recovery of textual data was done. They integrated two methods namely: ontology mastering from texts and reusing current terminological resources. The results obtained after processing through various steps were integrated with the Alzheimer's domain and its associated syndromes. 5765 concepts related to 7499 taxonomic and 10,889 non-taxonomic relationships were available in the system. Among these numbers, 439 ideas unavailable from the UMLS have been created and 608 new synonymous French terms had been added [36].

\section{ADIO}

In 2019, a group of researchers integrated the pre-existing ontology in AD to bring an increased accuracy of reasoning of the Alzheimer's disease. Alzheimer's disease integrated ontology (ADIO) combined two important biomedical ontology(a) Alzheimer's disease ontology (ADO) and (b) Alzheimer's disease map ontology (ADMO). OWL format was used to describe ADO. And the complexity of Alzheimer's disease pathophysiology was described by the ADMO. Since ADO and ADMO were relevant complements with each other, the satisfaction of knowledge resources of the Alzheimer's disease will be enhanced by their integration. The reliability and effectiveness of ADIO was checked by HermiT 1.4.3.456 reasoner in Protégé [37].

\section{ADO}

In 2014, researchers from Germany constructed ADO (Alzheimer's disease ontology) which contains information about preclinical, clinical, etiological, and molecular/ cellular concepts/categories with the aim of knowledge specific to AD with maximum coverage. Validation of this method has a satisfactory performance F score $=72 \%$. This ontology provides in depth ontological search in two different categories (a) searching 
concept-wise and (b) the synonyms associated with the concepts. It can also automatically retrieve specific knowledge related to the concepts of Alzheimer [38].

PredictND -The Prediction tool

The effectiveness of CDSS to predict the progression of subjective cognitive decline and mild cognitive impairment patients in memory clinics using PredictND tool was assessed. It consisted of 429 patients with subjective cognitive decline $(\mathrm{n}=230)$ and mild cognitive impairment $(\mathrm{n}=199)$ (females 54\%, age $67 \pm 9$, MMSE $28 \pm 2$ ) followed for at least 12 . The confidence level of the physician's prediction without and with the PredictND tool was assessed. $1.7 \pm 0.4$ years later, the overall prognostic accuracy was unaffected while using PredictND tool. However, the prognostic accuracy increased to $3 \%$ when restricted to patients with more certain classifications. Negative prediction value was also high. Overall, usage of PredictND tool significantly increased the confidence of prediction in prognosis of AD [39].

\section{Gene-ontology in Alzheimer's disease}

Gene ontology (GO) in Alzheimer's and dementia were created using GO terms to improve the neurological domains of the GO resource. It will benefit the dementia and Alzheimer's research community by providing GO more suitable for analyses of neurological datasets. It will benefit the dementia and Alzheimer's research community by providing GO more suitable for analyses of neurological datasets [40]. The GO databases were denoted as 'ARUK-UCL'. They collected GO data from dementiarelevant protein records; revised them and added new GO terms to them [41].

\section{IC-SMART}

IC-SMART, an IoTCloud depended Seamless Monitoring for Alzheimer diagnosis and Rehabilitation System (SMART) was built using semantic web particularly ontology for representing and organizing knowledge particular to AD. Information of the patient is gathered and the inputs of general practitioners and specialists were combined in the database for developing a Bayesian Network decision model to suggest diagnosis of $\mathrm{AD}$ patient. An android mobile based cloud software service was developed and implemented in the Amazon E2C cloud to verify its feasibility. Sensitivity analysis was also taken. The results of the prototype are very promising [42].

\section{Automatic ontology construction in Alzheimer's domain}

A completely automatic approach to develop ontology using ODP (ontology design patterns) and text corpora in Alzheimer's domain was proposed. It consists of learning ontology from texts followed by matching them with ODPs. The resulted ontology contains 381 terms and 184 relations. In addition to that 200 new words and about 42 new relations were integrated to them. The need for expert knowledge to check and remodel ontology is greatly reduced, and it will complete the process in a shorter duration of time [43].

\section{NDDO}

Neurodegenerative Disease Data Ontology (NDDO), an ontology developed from the pre-existing projects on ontology of data types (OntoDT) and ontology of core data mining entities (OntoDM-core) was developed representing a specific datatype in datasets of a particular domain. NDDO provides a semantic representation of datasets composed of diagnostic information of the neurodegenerative disease such as clinical 
presentation, images, biomarkers, and disease progression data of patients collected from the hospitals [44].

\section{CBR ontology for dementia caregiving}

Ontological CBR (Case Based Reasoning) system is a caregiver help tool. It can predict and detect dementia with the aid of an Ontological Case-based Learning System, known as DePicT Dementia Onto-CLASS provided the information of caring Tasks. Algorithm based on case retrieval is used in this system to give the correct result with increased accuracy. Hermit and myCBR evaluated the ontology and the obtained results are validated the caregivers [45].

\section{Mario}

Unfortunately, there is no cure for AD currently. Therapies to improve cognition of dementia people to stimulate and retain the functions and intellectual capabilities of the brain in addition the pharmacological treatment are found to be helpful. MARIO, an assistive robot that can support a set of knowledge intensive task was created with the aim of increasing autonomy and reducing loneliness in people with dementia and to support caregivers in their activity to assess patient's cognitive status. MARIO is a combination of robotics, ontology, and Semantic Web tech. Knowledge intensive tasks such as comprehensive geriatric assessment (CGA) and delivery of reminiscence therapy were performed by Mario. It was programmed with a set of pluggable software application. Its abilities were based on a common knowledge management framework such as its ability associated with retrieval of CGA questions to be asked to the patient from the framework and store the answers obtained from the patients and associated relevant metadata. It contains (a) a collection of interrelated and modularized ontologies, which represents all knowledge areas applicable to MARIO abilities, and (b) a set of software interfaces to provide high level approach to the ontology web system and the associated database. They also performed a demonstration on how the knowledge management framework supports the applications for CGA and reminiscence therapy, implemented on top of the knowledge base [17].

In [46], information related to the outdoor mobility of dementia patients were collected and organized to provide situation-aware assistance using assistive technology devices (ATDs). Here the knowledge base was deployed in the device which recognizes the environment/ situation of the patient and provide individual guidance in real time.

\section{Fuzzy logic}

In 2019, a project named EU neuGRID4You (N4U) generated various data sets from research labs and health centers and stored in a big data repository. Alzheimer's disease identification number (ADIN) was calculated using the big data and fuzzy logic through fuzzy processing. It enabled identifying patients in a particular intensity of $\mathrm{AD}$. This method and its decision relied on real data sets, demonstrators, and original neuroscience case studies. The results of 4 chosen case reports are promising with good understanding in patient disease information [47]. 
Paper-A systematic review of clinical decision support systems in Alzheimer's disease domain

Table 1. List of CDSS in Alzheimer's domain and the clinical recommendations

\begin{tabular}{|l|l|}
\hline \multicolumn{1}{|c|}{ CDSS in Alzheimer's Domain } & Clinical recommendations provided by each system \\
\hline Bayesian network based CDSS & Diagnosis of Alzheimer's disease \\
\hline Experience based CDSS & Diagnosis of Alzheimer's disease \\
\hline Cognitive and functional assessment based CDSS & Severity of Alzheimer's disease \\
\hline Dementia management and support system & $\begin{array}{l}\text { Diagnosis } \\
\text { Relevant laboratory investigation }\end{array}$ \\
\hline
\end{tabular}

\subsection{RQ5: Are there any ontology-based clinical decision support systems for} Alzheimer's disease?

\section{Ontology based CDSS in Alzheimer's disease}

Ontology in Alzheimer's domain represents and defines the information, properties, relationships of large quantity of data within that specific domain. The gathered information includes pathophysiology, preclinical, clinical, etiological, molecular, cellular and various other concepts within AD. Hence, a standardized and organized information of Alzheimer's domain can be maintained and used for various medical and research purposes.

To diagnose Alzheimer's disease, a large quantity of information acquired from the patient's examinations and test reports need to be processed and analyzed. For this purpose, three main ontologies are used in the development of this knowledge based CDSS [48]. (i)The MIND ontology suggests various clinical, laboratory and genetic tests performed for the diagnosis of AD. (ii) The SWAN ontology sets the criteria for diagnosis. (iii) The SNOMED CT ontology standardizes the data specific to Alzheimer's domain. A rule dependent reasoning model was used to diagnose AD over aforesaid knowledge base created by domain experts. This knowledge based CDSS model is validated in three hospitals for diagnosis of Alzheimer's disease. Results of the validation are yet to be released. Future projects include graphical ontology mapping tool, SOEKS (Set of Experience Knowledge Structure) and application of this model in other domains such as autism, cardiovascular diseases.

Using ontology and semantic web, a knowledge based CDSS was created to diagnose $\mathrm{AD}$. To diagnose $\mathrm{AD}$, multidisciplinary data were assembled, and reasoning was attained from the knowledge base. Ontologies used to build the knowledge base includes - SWAN, MIND ontology and SNOWMED CT. A rule dependent reasoning model was used to diagnose $\mathrm{AD}$ over aforesaid knowledge base created by domain experts. This knowledge based CDSS was verified by three hospitals.

Table 2. Ontology based projects in Alzheimer's domain

\begin{tabular}{|l|}
\hline \multicolumn{1}{|c|}{ ONTOLOGY BASED PROJECTS IN ALZHEIMER'S DOMAIN } \\
\hline ADO - Alzheimer's disease ontology \\
\hline ADIO - Alzheimer's disease integrative ontology \\
\hline ADMO - Alzheimer's disease map ontology \\
\hline NDDO - Neurodegenerative disease ontology \\
\hline GENE ONTOLOGY \\
\hline CBR ontology - Case based reasoning ontology
\end{tabular}




\section{Conclusion \& Future work}

Ontology based and non-ontology based CDSS in Alzheimer's domain aids physicians in clinical decision making. CDSS models developed in AD are found to be promising with a high accuracy rate. Ontology can process, analyze, and create semantic relationship of a huge amount of data. Hence an ontology based CDSS model that can suggest (i) relevant clinical, radiological, laboratory and genetic tests, (ii) diagnosis of Alzheimer's disease, (iii) staging and severity of the condition, (iv) prognosis, (v) treatment options, (vi) activities and exercises to promote neuroplasticity can be developed to aid the physicians throughout the patient care. The future works is to develop an ontology based CDSS in Alzheimer's domain with abilities to suggest tests, diagnosis, severity, prognosis, treatment, and neuroplasticity exercises is aspired.

\section{$5 \quad$ Acknowledgment}

The research leading to these results has received funding from the Research Council (TRC) of the Sultanate of Oman under the Block Funding Program BFP/ RGP/ ICT/ 19/ 229.

\section{References}

[1] “Alzheimer's disease," nhs.uk, May 10, 2018. https://www.nhs.uk/conditions/alzheimersdisease/ (accessed May 31, 2021).

[2] K. A, S. J, G. A, T. JW, and S. J, “Alzheimer Disease (Nursing),” Mar. 2021, Accessed: May 31, 2021. [Online]. Available: https://europepmc.org/article/nbk/nbk568805

[3] "10 Early Signs and Symptoms of Alzheimer's," Alzheimer's Disease and Dementia. https://alz.org/alzheimers-dementia/10_signs (accessed May 31, 2021).

[4] Erickson, K. I., Weinstein, A. M. and Lopez, O. L. "Physical Activity, Brain Plasticity, and Alzheimer's Disease," Arch. Med. Res., vol. 43, no. 8, pp. 615-621, Nov. 2012, https://doi.org/10.1016/j.arcmed.2012.09.008

[5] "Alzheimer disease in the United States (2010-2050) estimated using the 2010 census | Neurology." https://n.neurology.org/content/80/19/1778.short (accessed May 31, 2021). https://doi.org/10.1212/wnl.0b013e31828726f5

[6] "Alzheimer's Disease: Epidemiology, Risks, and Testing." https://www.rgare.com/ knowledge-center/media/articles/alzheimer-s-disease-epidemiology-risks-and-testing (accessed May 31, 2021).

[7] Alzheimer's Association, "2016 Alzheimer's disease facts and figures," Alzheimers Dement. J. Alzheimers Assoc., vol. 12, no. 4, pp. 459-509, Apr. 2016, https://doi.org/10.1016/ j.jalz.2016.03.001

[8] El-Metwally A. et al., (2019). "Epidemiology of Alzheimer's Disease and Dementia in Arab Countries: A Systematic Review," Behav. Neurol., vol., p. e3935943, Oct. 2019, https://doi.org/10.1155/2019/3935943

[9] “Alzheimers \& Dementia in Oman," World Life Expectancy. https://www.worldlifeexp ectancy.com/oman-alzheimers-dementia (accessed May 31, 2021). 
[10] "Dementia." https://www.who.int/news-room/fact-sheets/detail/dementia (accessed May $31,2021)$.

[11] "What Is Alzheimer's Disease? | National Institute on Aging." https://www.nia.nih.gov /health/what-alzheimers-disease (accessed May 31, 2021).

[12] "Epidemiology of Dementia and Alzheimer Disease in Individuals With Down Syndrome | Congenital Defects | JAMA Neurology | JAMA Network.” https://jamanetwork.com /journals/jamaneurology/article-abstract/2753237 (accessed May 31, 2021).

[13] Ferreira, D., Pereira, J. B., Volpe, G. and Westman, E. (2019). "Subtypes of Alzheimer's Disease Display Distinct Network Abnormalities Extending Beyond Their Pattern of Brain Atrophy," Front. Neurol., vol. 10, https://doi.org/10.3389/fneur.2019.00524

[14] “Alzheimer's disease - Symptoms and causes - Mayo Clinic.” https://www.mayoclinic.org /diseases-conditions/alzheimers-disease/symptoms-causes/syc-20350447 (accessed May 31, 2021).

[15] "Plasticity in Early Alzheimer's Disease: An Opportunity for Intervention." https://www.ncbi.nlm.nih.gov/pmc/articles/PMC3419487/ (accessed May 31, 2021).

[16] Bashir, S., Mizrahi, I., Weaver, K., Fregni, F. and Pascual-Leone, A. (2010). "Assessment and Modulation of Neural Plasticity in Rehabilitation With Transcranial Magnetic Stimulation," $P M \& R$, vol. 2, no. 12, Supplement, pp. S253-S268, Dec., https://doi.org/10.1016 li.pmrj.2010.10.015

[17] "Ontology-Based Knowledge Management for Comprehensive Geriatric Assessment and Reminiscence Therapy on Social Robots | Istituto di Scienze e Tecnologie della Cognizione." /en/content/ontology-based-knowledge-management-comprehensive-geriatricassessment-and-reminiscence (accessed May 31, 2021). https://doi.org/10.1007/978-3030-05249-2_6

[18] “Alzheimer's Symptoms: 24 Signs \& Symptoms of Alzheimer's Disease.” https://www. webmd.com/alzheimers/guide/understanding-alzheimers-disease-symptoms (accessed May 31, 2021).

[19] Etters, L., Goodall, D. and Harrison, B. E. (2008). "Caregiver burden among dementia patient caregivers: A review of the literature," J. Am. Acad. Nurse Pract., vol. 20, no. 8, pp. 423-428, https://doi.org/10.1111/j.1745-7599.2008.00342.x

[20] "An Overview of Clinical Decision Support System (CDSS) as a Computational Tool and Its Applications in Public Health | SpringerLink." https://link.springer.com/chapter /10.1007/978-3-030-35280-6_5 (accessed May 31, 2021).

[21] CDC, "How to Implement Clinical Decision Support Systems," Centers for Disease Control and Prevention, Jun. 25, 2020. https://www.cdc.gov/dhdsp/pubs/guides/bestpractices/clinical-decision-support.htm (accessed May 31, 2021).

[22] Carvalho, C. M., Seixas, F. L., Conci, A., Muchaluat-Saade, D. C., Laks, J. and Boechat, Y. (2020). "A dynamic decision model for diagnosis of dementia, Alzheimer's disease and Mild Cognitive Impairment," Comput. Biol. Med., vol. 126, p. 104010, https://doi.org/ $\underline{10.1016 / j . c o m p b i o m e d .2020 .104010 ~}$

[23] Seixas, F. L., Zadrozny, B., Laks, J., Conci, A. and Muchaluat-Saade, D. C. (2012). "A decision support system for Alzheimer's disease diagnosis"., Chapter II in: Scientific Computing Applied to Medicine and Healthcare, Eds. R.A. Feijoo, Ziviani A.and Blanco, P. J., MACC-LNCC, pp. 355-373.

[24] Laxmi, P., Gupta, D., Radhakrishnan, G., Amudha, J. and Sharma, K. (2021). "Automatic Multi-disease Diagnosis and Prescription System Using Bayesian Network Approach for Clinical Decision Making," in Advances in Artificial Intelligence and Data Engineering, Singapore, pp. 393-409. https://doi.org/10.1007/978-981-15-3514-7_31 
[25] Toro C. et al., (2012). "Using Set of Experience Knowledge Structure to Extend a Rule Set of Clinical Decision Support System for Alzheimer's Disease Diagnosis," Cybern. Syst., vol. 43, no. 2, pp. 81-95, https://doi.org/10.1080/01969722.2012.654070

[26] Lindgren, H. (2011). "Integrating Clinical Decision Support System Development into a Development Process of Clinical Practice - Experiences from Dementia Care," in Artificial Intelligence in Medicine, Berlin, Heidelberg, pp. 129-138. https://doi.org/10.1007 1978-3-642-22218-4_17

[27] Bucholc M. et al. (2019). "A practical computerized decision support system for predicting the severity of Alzheimer's disease of an individual," Expert Syst. Appl., vol. 130, pp. 157171, https://doi.org/10.1016/j.eswa.2019.04.022

[28] Carvalho, C. M., Seixas, F. L., Conci, A., Muchaluat-Saade, D. C., Laks, J. and Boechat, Y. (2020). "A dynamic decision model for diagnosis of dementia, Alzheimer's disease and Mild Cognitive Impairment," Comput. Biol. Med., vol. 126, p. 104010, https://doi.org/10. 1016/j.compbiomed.2020.104010

[29] "What are Ontologies? | Ontotext Fundamentals Series," Ontotext. https://www.onto text.com/knowledgehub/fundamentals/what-are-ontologies/ (accessed May 31, 2021).

[30] Shobowale, K. O. (2020). "Ontology in Medicine as a Database Management System," in Ontology-Based Information Retrieval for Healthcare Systems, John Wiley \& Sons, Ltd, pp. 69-90. https://doi.org/10.1002/9781119641391.ch4

[31] Gruber, T. R. (1995). "Toward principles for the design of ontologies used for knowledge sharing?,” Int. J. Hum.-Comput. Stud., vol. 43, no. 5, pp. 907-928, https://doi.org/10.1006/ ijhc. 1995.1081

[32] Zekri, F., Bouaziz, R. and Turki, E. (2015). "A fuzzy-based ontology for Alzheimer's disease decision support," in 2015 IEEE International Conference on Fuzzy Systems (FUZZIEEE), pp. 1-6. https://doi.org/10.1109/fuzz-ieee.2015.7337922

[33] Arisi I. et al., (2018). "An ontology-based approach to improve data querying and organization of Alzheimer's Disease data," in 2018 IEEE International Conference on Bioinformatics and Biomedicine (BIBM), pp. 2732-2734. https://doi.org/10.1109/bibm.2018.8621 $\underline{524}$

[34] Yamada D. B. et al., (2020). "Ontology-Based Inference for Supporting Clinical Decisions in Mental Health," in Computational Science - ICCS 2020, Cham, pp. 363-375. https://doi.org/10.1007/978-3-030-50423-6_27

[35] Gomez-Valadés, A., Martínez-Tomás, R. and Rincón-Zamorano, M. (2019). “Ontologies for Early Detection of the Alzheimer Disease and Other Neurodegenerative Diseases," in Understanding the Brain Function and Emotions, Cham, pp. 42-50. https://doi.org/ 10.1007/978-3-030-19591-5_5

[36] Dramé K. et al., (2014). "Reuse of termino-ontological resources and text corpora for building a multilingual domain ontology: An application to Alzheimer's disease," J. Biomed. Inform., vol. 48, pp. 171-182, https://doi.org/10.1016/j.jbi.2013.12.013

[37] hoaip, N., Barakat, S. and Elmogy, M. (2019). “Alzheimer's Disease Integrated Ontology (ADIO)," in 2019 14th International Conference on Computer Engineering and Systems (ICCES), pp. 374-379. https://doi.org/10.1109/icces48960.2019.9068176

[38] Malhotra, A., Younesi, E., Gündel, M., Müller, B., Heneka, M. T. and Hofmann-Apitius, M. (2014). "ADO: A disease ontology representing the domain knowledge specific to Alzheimer's disease," Alzheimers Dement., vol. 10, no. 2, pp. 238-246, https://doi.org/10. 1016/j.jalz.2013.02.009

[39] Bruun M. et al., (2019). "Impact of a clinical decision support tool on prediction of progression in early-stage dementia: a prospective validation study," Alzheimers Res. Ther., vol. 11, no. 1, p. 25, https://doi.org/10.1186/s13195-019-0482-3 
[40] "Gene Ontology: A Resource for Analysis and Interpretation of Alzheimer's Disease Data | Exon Publications.” https://exonpublications.com/index.php/exon/article/view/234 (accessed May 31, 2021).

[41] Kramarz B. et al., (2018). "Improving the Gene Ontology Resource to Facilitate More Informative Analysis and Interpretation of Alzheimer's Disease Data," Genes, vol. 9, no. 12, Art. no. 12, https://doi.org/10.3390/genes9120593

[42] Kaur P. D. and Sharma, P. (2020). "IC-SMART: IoTCloud enabled Seamless Monitoring for Alzheimer Diagnosis and Rehabilitation SysTem," J. Ambient Intell. Humaniz. Comput., vol. 11, no. 8, pp. 3387-3403, https://doi.org/10.1007/s12652-019-01534-5

[43] Cahyani D. E. and Wasito, I. (2017). "Automatic Ontology Construction Using Text Corpora and Ontology Design Patterns (ODPs) in Alzheimer's Disease," J. Ilmu Komput. Dan Inf., vol. 10, no. 2, Art. no. 2, https://doi.org/10.21609/jiki.v10i2.374

[44] Kostovska, A., Tolovski, I., Maikore, F., Soldatova, L. and Panov, P. (2019). "Neurodegenerative Disease Data Ontology," in Discovery Science, Cham, pp. 235-245. https://doi.org/10.1007/978-3-030-33778-0 19

[45] Nasiri, S., Zahedi, G., Kuntz, S. and Fathi, M. (2019). "Knowledge representation and management based on an ontological CBR system for dementia caregiving," Neurocomputing, vol. 350, pp. 181-194, https://doi.org/10.1016/j.neucom.2019.04.027

[46] Zhang, X., Hu, B., Ma, X., Moore, P. and Chen, J. (2014). "Ontology driven decision support for the diagnosis of mild cognitive impairment," Comput. Methods Programs Biomed., vol. 113, no. 3, pp. 781-791, https://doi.org/10.1016/j.cmpb.2013.12.023

[47] Munir, K., de Ramón-Fernández, A., Iqbal, S. and Javaid, N. (2019). "Neuroscience patient identification using big data and fuzzy logic-An Alzheimer's disease case study," Expert Syst. Appl., vol. 136, pp. 410-425, https://doi.org/10.1016/j.eswa.2019.06.049

[48] Sanchez E. et al., (2011). "A Knowledge-based Clinical Decision Support System for the diagnosis of Alzheimer Disease," in 2011 IEEE 13th International Conference on e-Health Networking, Applications and Services, pp. 351-357. https://doi.org/10.1109/health. $\underline{2011.6026778}$

\section{$7 \quad$ Authors}

Dr. Sherimon P.C. is working as Faculty of Computer Studies, Arab Open University, Muscat, Sultanate of Oman. He has Ph.D. in Computer Applications from Manonmaniam Sundaranar University, Tamil Nadu, India. He won the first National Research award from TRC (The Research Council), Oman in Information and Communication Technology sector. He has published several articles in International Journals and Conference Proceedings. His research interests lie in semantic web, ontology, and big data.

Dr. Vinu Sherimon is working in Department of IT, University of Technology and Applied Sciences, Muscat, Sultanate of Oman. She has Ph.D. in Computer Science from Manonmaniam Sundaranar University, Tamil Nadu, India. She has published several articles in International Journals and Conference Proceedings. Her research interests lie in blockchain, semantic web, pervasive computing, ontology, big data, and data mining.

Preethii S.P. is a pre-final year M.B.B.S. student in Government Medical College, Vellore, Tamil Nadu, India. 
Dr. Rahul V. Nair is a Grade A medical officer in Department of Accident and Emergency, Royal Oman Police (ROP) Hospital, Muscat, Sultanate of Oman. He received M.B.B.S. from K.S. Hegde Medical Academy, Mangalore, India. He has won ICMR Research Work Award on the topic "Relationship between Vitamin-C blood level and Hypertension". His research interests include machine learning and deep learning.

Dr. Renchi Mathew is a senior Consultant in Department of General Medicine, Royal Oman Police (ROP) Hospital, Muscat, Sultanate of Oman. In 2009, he received FRCP from the Royal College of Physicians and Surgeons of Glasgow, Scotland.

Article submitted 2021-04-30. Resubmitted 2021-05-19. Final acceptance 2021-05-20. Final version published as submitted by the authors. 